\title{
Propriedades Psicométricas da Versão Revisada da Dimensão Necessidade de Atenção do Inventário Dimensional Clínico da Personalidade
}

\author{
Lucas de Francisco Carvalho ${ }^{1}$ \\ Programa de Pós Graduação Stricto Sensu em Psicologia da Universidade São Francisco, \\ Itatiba, São Paulo, Brasil \\ Catarina Possenti Sette \\ Curso de Psicologia da Universidade São Francisco, Itatiba, São Paulo, Brasil \\ Cláudio Garcia Capitão \\ Ricardo Primi \\ Programa de Pós Graduação Stricto Sensu em Psicologia da Universidade São Francisco, \\ Itatiba, São Paulo, Brasil
}

\begin{abstract}
Resumo
A presente pesquisa teve como objetivo investigar as propriedades psicométricas da versão revisada da dimensão Necessidade de Atenção do Inventário Dimensional Clínico da Personalidade (IDCP). Para isso foram 120 participantes compondo o estudo, com idade variando entre 17 e 54 anos $(M=24,6$; $D P=7,96)$, sendo 96 mulheres $(80 \%)$. Todos os sujeitos responderam o IDCP, o Inventário de Personalidade NEO-PI Revisado e um questionário de autoavaliação desenvolvido para este estudo. Os dados encontrados no estudo foram favoráveis em relação às evidencias de validade com base na estrutura interna, apresentando quatro componentes teoricamente coerentes e interpretáveis; evidência de validade com base na relação com outras variáveis, indicando relações coerentes entre o escore total e componentes da nova dimensão e variáveis externas ao instrumento; e quanto à fidedignidade por consistência interna da dimensão revisada e seus componentes (entre 0,73 e 0,86 ). Nesse sentido, conclui-se que a dimensão revisada é mais adequada da perspectiva psicométrica se comparada com sua versão original, além de estar mais relacionada ao funcionamento patológico da personalidade.
\end{abstract}

Palavras-chave: Transtornos psiquiátricos, DSM, personalidade.

\section{Psychometric Properties of the Revised Attention Seeking Dimension of the Inventário Dimensional Clínico da Personalidade}

\begin{abstract}
The present study aimed to investigate the psychometric properties of the revised scale Need for Attention of the Inventário Dimensional Clínico da Personalidade (IDCP). 120 participants composed the study, aged between 17 and 54 years $(M=24.6 ; S D=7.96)$ and 96 women $(80 \%)$. All subjects answered the IDCP, the NEO Personality Inventory-Revised and a self-assessment questionnaire developed for
\end{abstract}

Endereço para correspondência: Universidade São Francisco, Rua Alexandre Rodrigues Barbosa, 45, Campus de Itatiba, Itatiba, SP, Brasil 13251-900. E-mail: lucas@labape.com.br, caty_sette@hotmail.com, claudio. capitao@saofrancisco.edu.bre rprimi@mac.com Fomento: Conselho Nacional de Desenvolvimento Científico e Tecnológico (CNPq); Fundação de Amparo à Pesquisa do Estado de São Paulo (FAPESP). 
this study. The findings in the study were favorable in relation to evidence of validity based on internal structure, featuring four components theoretically coherent and interpretable; validity evidence based on relations with other variables, indicating coherent relationships between the total score and components of the new dimension with external variables to the instrument; and reliability (internal consistency) of the scale reviewed and its components (between .73 and .86). Accordingly, it is concluded that the reviewed scale is more adequate from the psychometric perspective compared to its original version, and is more related to pathological personality functioning.

Keywords: Psychiatric disorders, DSM, personality.

\section{Propiedades Psicométricas de la Versión Revisada de la Dimensión Necesidad de Atención del Inventário Dimensional Clínico da Personalidade}

\section{Resumen}

El presente estudio tuvo como objetivo investigar las propiedades psicométricas de la escala revisada Necesidad de Atención del Inventário Dimensional Clínico da Personalidade (IDCP). Para ello fueron 120 los participantes que componen el estudio, de edades comprendidas entre 17 y 54 años $(M=24.6$, $S D=7.96)$ y 96 mujeres ( $80 \%)$. Todos los sujetos respondieron el IDCP, el Inventario de Personalidad NEO-PI revisado y um cuestionario de autoevaluación desarrollado para este estudio. Los hallazgos del estudio fueron favorables en relación con la evidencia de la validez basada en la estructura interna, con cuatro componentes teóricamente coherente e interpretable; evidencia de validez basada en las relaciones con otras variables, indicando relaciones coherentes entre la puntuación total y los componentes de la nueva dimensión; y las variables externas en el instrumento, así como de la fiabilidad (consistencia interna) de la escala revisada y sus componentes (entre .73 y .86). En consecuencia, se concluye que la escala revisada en la perspectiva psicométrica más adecuada en comparación con su versión original, además de estar más relacionado con funcionamiento de la personalidad patológica.

Palabras clave: Trastornos psiquiátricos, DSM, la personalidad.

A personalidade é compreendida como um padrão de características inter-relacionadas, constantes que são frequentemente não conscientes e quase automáticas, apresentadas nos ambientes típicos de um determinado indivíduo (Millon, Millon, Meagher, Grossman, \& Ramanath, 2004). Em outras palavras, trata-se de um conjunto de características mais persistentes e salientes apresentadas por um indivíduo para enfrentar o cotidiano (Carvalho, 2008).

Millon et al. (2004) considera a personalidade como uma malha entrelaçada de estruturas estáveis (memórias internalizadas e autoimagens) e funções coordenadoras (processos cognitivos e mecanismos inconscientes), tendo como ponto de partida a visão de homem integrado. Nesse contexto teórico, esse construto é compreendido como estilos, padrão de reações presentes e persistentes, mais ou menos adaptativos apresentados pelos indivíduos frente às situações. São diversos os fatores que contribuem para o desenvolvimento da personalidade, podendo ser biológicos, psicológicos ou sociais, porém, o mais importante em seu desenvolvimento é a interação desses fatores (Millon, 2011; Millon \& Davis, 1996; Millon et al., 2004).

A noção de personalidade, tanto em seu desenvolvimento saudável quanto no patológico, implica uma ideia da personalidade em um continuum. Por um lado, o funcionamento psicológico pode apresentar-se de forma mais saudável com a presença de características que são mais comuns entre outras pessoas, relacionadas à autonomia, à eficácia nas competências, a uma tendência a ajustar-se efetivamente ao meio social, a um senso subjetivo de satisfação e a uma 
habilidade de colocar em uso suas potencialidades. Por outro, o funcionamento psicológico pode apresentar-se de maneira mal-adaptativa ou patológica, podendo acarretar uma dificuldade na convivência do indivíduo nos diferentes ambientes.

Em alguns casos, o funcionamento patológico da personalidade pode configurar-se como um transtorno da personalidade que pode ser compreendidos como representações de diversos padrões em que a personalidade funciona de maneira mal-adaptada em relação ao ambiente, acarretando prejuízos importantes na vida dessas pessoas (Millon, 1993; Millon, Grossman, \& Tringone, 2010; Widiger \& Trull, 2007).

Dentre as diversas teorias da personalidade presentes na literatura, é provável que uma das escassas abordagens que se propõe a integrar teoria, avaliação e intervenção dos transtornos da personalidade seja o modelo desenvolvido por Millon (Millon \& Davis, 1996; Strack \& Millon, 2007), além de apresentar respaldo empírico, evidenciados com base no Millon Clinical Multiaxial Inventory (MCMI; Choca, 2004; Millon \& Davis, 1997; Millon, Millon, \& Davis, 1994; Strack \& Millon, 2007). De acordo com Loureiro (2000), a proposta de Millon se encaixa no que atualmente é compreendido como adequado em relação a uma abordagem completa da personalidade e seus transtornos, ou seja, considera a personalidade em seus diferentes níveis e dimensões.

O presente estudo tem como base a teoria de Millon, principalmente em relação às características que dificultam a adaptação do sujeito em seu respectivo ambiente, o que pode caracterizar um transtorno da personalidade. Millon et al. (2004) propõem quatorze estilos patológicos da personalidade, que são: esquizoide, evitativo, depressivo, dependente, histriônico, narcisista, antissocial, sádico, compulsivo, negativista, masoquista, paranoide, esquizotípico e borderline. Em referência mais atual (Millon, 2011), é também proposto o estilo hipomaníaco.

Com base na proposta de Millon, bem como nos estilos derivados, e no eixo do DSM-IV-TR, Carvalho e Primi (2011) desenvolveram, no Brasil, o Inventário Dimensional Clínico da Perso- nalidade (IDCP). Trata-se de um instrumento para a avaliação dos aspectos patológicos da personalidade, composto por 163 itens distribuídos em 12 dimensões, sendo elas: Dependência, Agressividade, Instabilidade de Humor, Excentricidade, Necessidade de Atenção, Desconfiança, Grandiosidade, Desapego, Evitação Social, Autossacrificio, Conscienciosidade e Impulsividade. Aplicando-se o instrumento, é possível obter os perfis de dimensões da personalidade do sujeito avaliado, sendo que altas pontuações sugerem características tendenciosas a um funcionamento mais patológico da personalidade (Carvalho, 2011).

Em relação às propriedades psicométricas das dimensões do IDCP, os dados exibidos por Carvalho (2011) demonstram a adequação em relação aos índices de fidedignidade e evidências de validade (com base na estrutura interna e na relação com variáveis externas). O IDCP foi aplicado em 1281 pessoas (1154 sem diagnóstico psiquiátrico conhecido e 127 pacientes com diagnóstico de transtornos psiquiátricos) com idade variando entre 18 e 90 anos $(M=26,64$; $D P=8,94)$, sendo $61,8 \%$ do sexo feminino. Posteriormente, foram realizadas análises fatoriais exploratórias e confirmatórias e verificação dos coeficientes de fidedignidade por meio do alfa de Cronbach, sendo que este índice se mostrou satisfatório para onze dimensões completas do instrumento, considerando como ponto de corte 0,70 (Nunnally, 1978). Também foram verificadas evidencias de validade com base na relação com outras variáveis, particularmente, com as dimensões e facetas do Inventário de Personalidade NEO-PI Revisado (NEO-PI-R) e com diagnósticos psiquiátricos. De uma forma geral, foram encontradas as relações previamente esperadas entre as dimensões do IDCP e facetas e dimensões do NEO-PI-R, bem como com os diferentes diagnósticos psiquiátricos do eixo II do DSM-IV-TR (American Psychiatry Association [APA], 2002). Carvalho (2011) sugere algumas recomendações para reformulação no instrumento, entre elas, o desenvolvimento de novos itens para a dimensão Necessidade de Atenção, bem como a reformulação de alguns itens existentes dessa dimensão. 
Foi observada uma tendência de maior endosso aos itens dessa dimensão em relação à maior parte das dimensões do IDCP (Carvalho, 2011), de modo que indivíduos sem histórico de diagnóstico tenderam a apresentar altas pontuações. Nesse sentido, os resultados encontrados configuram-se como uma dificuldade para a validade da dimensão Necessidade de Atenção, pois sugere baixa discriminação entre os sujeitos que apresentam funcionamentos patológicos da personalidade e os sujeitos com funcionamentos mais saudáveis.

A literatura tratando da avaliação de características patológicas da personalidade aponta para a problemática dos instrumentos de autorrelato nesse contexto, considerando que a maior parte deles está voltada tipicamente para avaliação de características saudáveis da personalidade (Tomiatti, Gore, Lynam, Miller, \& Widiger, 2011). Entre as características patológicas da personalidade, aquelas relacionadas ao transtorno da personalidade histriônico estão entre as mais problemáticas, como apontado na literatura (Miller, 2012; Samuel \& Widiger, 2008).

É possível que uma das dificuldades na avaliação das características desse transtorno esteja relacionada com a proximidade entre essas e características que são esperadas e socialmente reforçadas. Uma explicação para essa tendência à avaliação de características mais saudáveis do que patológicas relacionadas à Necessidade de Atenção, esta em sua própria definição, referindo-se a uma necessidade exagerada de ter atenção dos outros, utilizando-se de mecanismos de sedução, reações exageradas e busca intensa por amizades. De acordo com Millon et al. (2004), essas características são socialmente desejadas do ponto de vista da sociedade. Por isso, é importante que ferramentas para avaliação de características histriônicas sejam capazes de avaliar níveis mais patológicos do construto.

Tomando como base os dados expostos e a importância de garantir que todas as dimensões do IDCP avaliem efetivamente as características patológicas da personalidade, Carvalho, Sette, Capitão e Primi (2013) realizaram uma pesquisa de revisão da dimensão Necessidade de Atenção. No estudo, foi utilizada a literatura acerca dos construtos relacionados a essa dimensão e também os conteúdos tipicamente relacionados ao transtorno da personalidade histriônico (Millon, 2011), como comportamentos de sedução e manipulação, busca ativa pela atenção do outro, entre outros. O estudo foi dividido em seis etapas distintas, descritas a seguir.

A primeira etapa caracterizou-se pelo levantamento bibliográfico para verificação das características relacionadas ao construto Necessidade de Atenção e ao transtorno da personalidade histriônico. Considerou-se, para tanto, as publicações pertencentes à quinta edição do DSM (DSM-5; APA, 2012); as dimensões componentes do Personality Inventory for DSM 5 (PID-5; Krueger, Derringer, Markon, Watson, \& Skodol, 2011) as dimensões avaliadas pelo Shedler-Westen Assessment Procedure-200 (SWAP-200; Shedler \& Westen, 2004); e, as dimensões propostas por Anna Clark (1990). Na etapa seguinte, foi realizada a seleção, com base na literatura citada, das características relacionadas à dimensão Necessidade de Atenção do IDCP, organizando-os em uma planilha eletrônica com as respectivas traduções e adaptações culturas realizadas pelos pesquisadores.

$\mathrm{Na}$ terceira etapa, foi realizada a operacionalização dos construtos selecionados, ou seja, desenvolvimento de itens para todas as características presentes na planilha. Estes itens foram baseados em 17 construtos selecionados na etapa anterior. $\mathrm{O}$ número de itens desenvolvidos para cada um dos construtos se deu em função da quantidade de informação inicial obtida pelos pesquisadores acerca desses construtos. $\mathrm{Na}$ quarta etapa, foram selecionados os itens considerados mais adequados.

A representatividade dos 32 itens elaborados e selecionados em relação aos itens originais da dimensão foi verificada na quinta etapa. $\mathrm{E}$, por último, na etapa 6 , foi feita a seleção final dos novos itens para a composição da versão revisada da dimensão Necessidade de Atenção e a revisão final dos itens selecionados ao longo das etapas, considerando, sobretudo, a etapa 5 . Como produto final, a nova dimensão Necessidade de Atenção ficou composta por 48 novos itens, sendo 16 itens provenientes da versão original da dimensão. 
Considerando a elaboração de um novo conjunto de itens para avaliação da dimensão Necessidade de Atenção do IDCP, o objetivo do presente estudo é investigar as propriedades psicométricas da versão revisada da dimensão $\mathrm{Ne}$ cessidade de Atenção do IDCP. Para tanto, foram verificadas evidências de validade com base na estrutura interna e na relação com variáveis externas, bem como os índices de fidedignidade (consistência interna) para a dimensão.

Em relação às variáveis externas, foram utilizadas as dimensões Neuroticismo, Agradabilidade e Extroversão do Inventário de Personalidade NEO PI Revisado (Costa \& McCrae, 2009). O instrumento foi escolhido por ser da mesma natureza que o IDCP (autorrelato) e pela robustez empírica do modelo implícito (Modelo dos Cinco Grandes Fatores), mesmo a literatura sugerindo algumas dificuldades do instrumento na mensuração das características patológicas da personalidade (Lynam, 2012; Miller, 2012; Samuel \& Gore, 2012; Widiger \& Costa, 2012). Também as dimensões foram escolhidas pela proximidade apontada pela literatura com o funcionamento histriônico (Widiger \& Lowe, 2008), intimamente relacionado à dimensão $\mathrm{Ne}$ cessidade de Atenção.

\section{Método}

\section{Participantes}

Participaram desta pesquisa 120 sujeitos, acessados por conveniência em uma universidade particular do interior do Estado de São Paulo, com idade variando entre 17 e 54 anos $(M=24,6$; $D P=7,96)$, sendo 96 mulheres $(80 \%)$. A maior parte apresentou ensino superior incompleto $(81,6 \%)$. Em relação à realização de tratamento psiquiátrico e/ou uso de medicamento psicotrópico, 14 sujeitos responderam que já fizeram tratamento psiquiátrico e apenas 3 participantes já utilizaram medicação. Além disso, 30 sujeitos disseram já ter feito tratamento psicológico e 12 ainda fazem psicoterapia.

\section{Instrumentos}

Foi utilizado o Inventário Dimensional Clínico da Personalidade, desenvolvido em 2011 por Carvalho e Primi, baseando-se na teoria de Millon e nos critérios diagnósticos das categorias apresentadas no eixo II do DSM-IV-TR. O instrumento é composto por 12 dimensões da personalidade, sendo elas: dependência, agressividade, instabilidade de humor, excentricidade, necessidade de atenção, desconfiança, grandiosidade, isolamento, evitação à crítica, autossacrifício, Conscienciosidade e impulsividade. Os itens estão dispostos em uma escala Likert de 1 a 4 pontos, sendo que 1 corresponde a não tem nada a ver comigo e 4 tudo a ver comigo. $\mathrm{O}$ tempo médio para execução do instrumento é de 25 minutos.

A partir da aplicação do instrumento, é possível a obtenção de perfis de dimensões da personalidade do indivíduo que é submetido à avaliação, de forma que altas pontuações propõem características tendenciosas a um funcionamento mais patológico da personalidade (Carvalho, 2011). Em relação às propriedades psicométricas das dimensões do IDCP, os dados exibidos por Carvalho (2011) demonstram a adequação em relação aos índices de fidedignidade e evidências de validade (com base na estrutura interna e na relação com variáveis externas), como já referido anteriormente. Foram realizadas análises fatoriais exploratórias e confirmatórias e verificação dos coeficientes de fidedignidade por meio do alfa de Cronbach, sendo que este índice se mostrou satisfatório para onze dimensões completas do instrumento, considerando como ponto de corte 0,70 (Nunnally, 1978). Também foram verificadas evidencias de validade com base na relação com outras variáveis, particularmente, com as dimensões e facetas do Inventário de Personalidade NEO-PI Revisado (NEO-PI-R) e com diagnósticos psiquiátricos. De uma forma geral, foram certificas as relações previamente esperadas entre as dimensões do IDCP e facetas e dimensões do NEO-PI-R, bem como com os diferentes diagnósticos psiquiátricos do eixo II do DSM-IV-TR (APA, 2002). Ressalta-se que, no caso do NEO-PI-R, as facetas são subgrupos de itens das dimensões.

Também foi aplicada a versão brasileira do Inventário de Personalidade NEO Revisado (NEO-PI-R; Costa \& McCrae, 2009). Trata-se de um inventário de autorrelato, composto por 
240 itens, cujo objetivo é a avaliação psicológica da personalidade de adultos em cinco dimensões: Neuroticismo, Extroversão, Abertura à Experiência, Amabilidade e Conscienciosidade. O instrumento deve ser respondido em uma escala do tipo Likert de 5 pontos, que vão de "discordo fortemente" (1) até "concordo fortemente" (5). Para este estudo, porém, somente foram consideradas as dimensões Neuroticismo, Extroversão e Agradabilidade. O tempo de aplicação para esse instrumento é de aproximadamente 40 minutos. O manual da versão brasileira do instrumento apresenta diversos estudos demonstrando evidências de validade e índices de fidedignidade satisfatórios (Costa \& McCrae, 2009).

Ainda, foi aplicado um questionário de autoavaliação desenvolvido pelos autores desta pesquisa contendo nove perguntas relacionadas com os construtos levantados na literatura, na primeira etapa do estudo. Para este estudo, foram utilizadas cinco das nove perguntas, somente as de caráter quantitativo, sendo elas, "quantas vezes você costuma ir a eventos sociais por semana?", "quantas vezes você costuma comprar roupas para você mesmo por semana?", "quanto você se importa com a roupa que usa?", "quantos relacionamentos amorosos você teve?" e "quantos amigos (de ambos os sexos) íntimos/próximos você tem?". A elaboração das perguntas para o questionário foi baseada nos comportamentos típicos de sujeitos com funcionamento histriônico de acordo com Millon (2011), e buscou-se selecionar comportamentos e atitudes pouco passíveis de subjetividade, aproximando-se ao máximo do que se espera para critérios externos (American Educational Research Association [AERA], APA, \& National Council on Measurement in Education [NCME], 1999). Ainda assim, não foram realizados estudos verificando as propriedades psicométricas desse questionário.

\section{Procedimentos e Análise dos Dados}

Em um primeiro momento esta pesquisa foi submetida a um Comitê de Ética em Pesquisa e após ser aprovada (CAAE 0144.0.142.000-07) iniciou-se a coleta. A aplicação foi realizada de forma coletiva dentro de salas de aula, em uma única sessão para cada turma, sendo necessário, em média, 40 minutos, bem como de forma individual, nos casos dos participantes que se encontravam fora da universidade. Primeiro foi explicado os objetivos da pesquisa e após os sujeitos consentirem em participar do estudo os mesmos assinaram o Termo de Consentimento Livre e Esclarecido e em seguida responderam aos instrumentos da pesquisa.

Após a coleta, os dados foram tabulados e procedeu-se às análises estatísticas. Considerando os objetivos deste estudo, primeiramente, o número de componentes a serem mantidos na análise por componentes principais foi verificado com base na análise paralela (Hayton, Allen, \& Scarpello, 2004; Watkins, 2006). Para veiculação da análise, foi utilizado o software R versão 2.15.3, uma vez que o mesmo possibilita o uso da análise paralela para variáveis policóricas, como é caso do presente estudo.

A partir disso, foi gerado um banco de dados para o software MPlus versão $6.12 \mathrm{com}$ o intuito de realizar a análise por componentes principais com variáveis policóricas, além da obtenção de índices confirmatórios que indicam a adequação da estrutura encontrada com base na presente amostra. Ressalta-se, ainda, que o ajustamento nos índices confirmatórios também sugere a adequação da amostra para a realização da análise. Os dados encontrados foram comparados com os dados apresentados por Carvalho (2011). Por fim, os componentes encontrados, dos construtos relacionados à dimensão Necessidade de Atenção, foram correlacionados com as três dimensões do NEO-PI-R citadas anteriormente.

\section{Resultados e Discussão}

A partir da análise paralela para variáveis policóricas, foram obtidos até 4 componentes com eigenvalue expressivos não evidenciados ao acaso. Em seguida, procedeu-se à análise por componentes principais via software MPLUS, forçando-se a solução de 4 componentes, utilizando a rotação oblíqua geomin, e método de extração Maximum Likelihood (ML), considerado como adequado para variáveis policóricas. Ini- 
cialmente foram analisados os índices de ajuste gerados, sendo, $\mathrm{X}^{2} / d f=1,22$; RMSEA $=0,045$; $\mathrm{CFI}=0,922 ; \mathrm{e}, \mathrm{SMR}=0,087$. Considerando-se os pontos de corte (Hooper, Coughlan, \& Mullen, 2008), os índices de ajuste foram bons $\left(\mathrm{X}^{2} / d f\right.$, RMSEA e SMR) e aceitáveis (CFI). Considera-se a partir dos dados apresentados a manuten- ção da estrutura de quatro componentes a partir da amostra utilizada nesta pesquisa.

As cargas fatoriais encontradas, o número de itens mantidos por componente e os índices de consistência interna (alfa de Cronbach) podem ser visualizados na Tabela 1. Ainda, os itens que permaneceram em cada um dos componentes estão em negrito.

Tabela 1

Análise Fatorial Exploratória e Índices de Consistência Interna

\begin{tabular}{ccccc}
\hline Item & $\begin{array}{c}\text { Sedução } \\
\text { e Manipulação }\end{array}$ & Intensidade Emocional & Busca por Atenção & $\begin{array}{c}\text { Superficialidade } \\
\text { Interpessoal }\end{array}$ \\
\hline 1 & $\mathbf{0 , 8 0 2}$ & 0,112 & 0,266 & 0,363 \\
2 & $\mathbf{0 , 7 7 0}$ & 0,294 & 0,369 & 0,268 \\
5 & $\mathbf{0 , 7 2 5}$ & 0,096 & 0,067 & 0,207 \\
22 & 0,456 & $\mathbf{0 , 7 9 2}$ & 0,229 & 0,264 \\
24 & 0,408 & 0,058 & $\mathbf{0 , 6 6}$ & 0,310 \\
27 & 0,349 & 0,073 & 0,126 & $\mathbf{0 , 6 7 4}$ \\
29 & 0,315 & 0,035 & 0,267 & $\mathbf{0 , 8 3 0}$ \\
31 & 0,300 & $\mathbf{0 , 8 3 4}$ & 0,163 & 0,238 \\
33 & 0,280 & 0,061 & $\mathbf{0 , 7 0 9}$ & 0,137 \\
34 & 0,266 & 0,207 & 0,197 & $\mathbf{0 , 9 0 3}$ \\
40 & 0,218 & 0,488 & $\mathbf{0 , 5 1 1}$ & 0,196 \\
41 & 0,191 & 0,348 & $\mathbf{0 , 6 5 9}$ & 0,163 \\
44 & 0,116 & $\mathbf{0 , 7 5 9}$ & 0,426 & 0,127 \\
№ Itens & 3 itens & 3 itens & 4 itens & 3 itens \\
A & 0,79 & 0,81 & 0,73 & 0,86 \\
\hline
\end{tabular}

Nota. Estão apresentados nas tabelas somente os itens que foram mantidos, facilitando a visualização dos dados.

Pode-se observar na Tabela 1 que foi selecionado um total de 13 itens para a nova dimensão Necessidade de Atenção. É importante ressaltar que buscou-se explicitamente manter um número mínimo de itens por componente encontrado, de modo a não impossibilitar o uso do instrumento pelos profissionais por conta de sua extensão. Desse modo, alguns itens que apresentaram carga fatorial adequada nos componentes não permaneceram. Basicamente, foram utilizados três critérios para a exclusão dos itens, sendo eles, (a) o item prejudicar ou não favorecer a consistência interna do componente, (b) pouca consistência interpretativa para manutenção do item no componente, (c) cargas expressivas em outros componentes. Com base nesses critérios, e no critério geral dizendo respeito a manutenção do menor número possível de itens, chegou-se ao conjunto final de itens para a versão revisada da dimensão. Vale ressaltar que entre os itens excluídos, alguns poderiam ser mantidos do ponto de vista psicométrico e mesmo de conteúdo. Contudo, considerando o número de dimensões do instrumento, um número excessivo de itens para o IDCP tornaria inviável sua aplicação. Necessidade de Atenção, variando 
entre 3 e 4 itens por componente. Em relação à consistência interna dos componentes encontrados, verifica-se uma variação entre 0,73 e 0,86 , sendo que a dimensão obteve índice de 0,83 . Os índices encontrados são considerados pela literatura como adequados, sobretudo, considerado o número pequeno de itens por componente (Embretson, 1996; Nunnally, 1978).

O primeiro componente chamado de Sedução e Manipulação agrupa itens relacionados a comportamentos de manipulação para ter atenção das pessoas, utilizando-se muitas vezes da sedução (exemplo de item, "Consigo seduzir as pessoas com facilidade"). O segundo componente, Intensidade Emocional, se refere a itens sobre uma crença em ter sentimentos mais extremos que a maioria das pessoas e da necessidade em demonstrá-los aos outros (exemplo de item, "Minhas emoções são sempre muito extremas").

O terceiro componente denominado Busca por atenção está relacionado a uma necessidade importante em ser o centro das atenções, como também a necessidade de estar sempre entre pessoas e ter muitos amigos (exemplo de item, "Não há nada melhor do que ter a atenção das pessoas"). O último componente, Superficialidade Interpessoal, agrupa itens que se referem à crença do indivíduo em conseguir em pouco tempo e com facilidade estabelecer vínculos íntimos com pessoas (exemplo de item, "Em pouco tempo consigo conhecer muito sobre as pessoas a ponto de nos tornarmos amigos"). Com base nessas descrições, foi verificada a representatividade dos modelos teóricos utilizados como base para o desenvolvimento da nova dimensão em cada um dos componentes encontrados (para detalhes acerca dos modelos, vide Carvalho et al., 2013).

A análise indicou que as propostas de acordo com o Personality Inventory for DSM 5 (DSM-5; Krueger et al., 2011), Shedler Westen Assessment Procedure (SWAP; Shedler \& Westen, 2004) e Clark (1990) foram utilizadas como base para itens selecionados para o conjunto final que representa a nova dimensão, apresentado neste estudo. Os componentes Sedução e Busca por Atenção são compostos por itens com base no PID-5 e SWAP; Intensidade Emocional por itens com base no PID-5 e Clark (1990); e, Su- perficialidade Interpessoal por itens com base em Clark. Observa-se também que nenhum dos itens foi baseado diretamente na proposta do DSM-5. Isso porque a proposta para o DSM5 (APA, 2012) exclui a categoria diagnóstica transtorno da personalidade histriônico, que está tipicamente relacionada com a dimensão foco desta pesquisa. Ainda assim, vale a pena ressaltar que o PID-5 exprime também a proposta para o DSM-5 (vide Krueger et al., 2011). Especificamente no que se refere à proposta para o DSM5 de exclusão do referido diagnóstico, a mesma não é consensual na literatura, o que pode ser ultimamente visualizado pela não aprovação da proposta no manual publicado (APA, 2013).

Uma vez que a estrutura interna do instrumento tenha sido definida, na continuidade os componentes da dimensão e seu escore total foram relacionados e comparados com variáveis externas. Na Tabela 2 os resultados da correlação são apresentados.

É possível observar que praticamente todas as magnitudes de correlação apresentadas foram significativas e positivas. Além disso, o grupo de itens da versão original da dimensão demonstrou estar mais relacionado com os componentes Sedução e Superficialidade Interpessoal, sugerindo que a nova dimensão conseguiu abranger características relacionadas ao construto necessidade de atenção não contemplados pela dimensão original, sobretudo, as características dos componentes Intensidade Emocional e Busca por Atenção. Por fim, os componentes apresentaram magnitudes de correlação baixas e moderadas entre si, indicando que indivíduos com pontuações altas ou baixas no escore total da nova dimensão podem apresentar discrepâncias entre os componentes que compõe a dimensão, ou seja, considerar os componentes é mais informativo do perfil do sujeito em relação a considerar apenas o escore total da dimensão. Também na Tabela 2 estão apresentadas as magnitudes de correlação entre os subcomponentes da nova dimensão Necessidade de Atenção e as dimensões do NEO-PI-R.

Pode-se observar que todas as magnitudes de correlação estatisticamente significativas com as dimensões Neuroticismo e Extroversão foram 
Tabela 2

Correlações entre os Escores Totais, Componentes e Dimensões do NEO-PI-R

\begin{tabular}{|c|c|c|c|c|c|c|c|c|c|}
\hline & 1 & 2 & 3 & 4 & 5 & 6 & Neuro & Extro & Agrad \\
\hline $\begin{array}{l}\text { 1. Necessidade de } \\
\text { Atenção (Revisada) }\end{array}$ & 1 & $0,70^{* *}$ & $0,68^{* *}$ & $0,69^{* *}$ & $0,68^{* *}$ & $0,62^{* *}$ & $0,38^{* *}$ & $0,41^{* *}$ & $-0,37^{* *}$ \\
\hline $\begin{array}{l}\text { 2. Necessidade de } \\
\text { Atenção (Original) }\end{array}$ & $0,70^{* *}$ & 1 & $0,65^{* *}$ & $0,32^{* *}$ & $0,31^{* *}$ & $0,63^{* *}$ & 0,01 & $0,60^{* *}$ & $-0,17$ \\
\hline 3. Sedução & $0,68^{* *}$ & $0,65^{* *}$ & 1 & $0,29^{* *}$ & $0,24^{* *}$ & $0,36^{* *}$ & 0,08 & $0,28^{* *}$ & $-0,35^{* *}$ \\
\hline $\begin{array}{l}\text { 4. Intensidade } \\
\text { Emocional }\end{array}$ & $0,69^{* *}$ & $0,32^{* *}$ & $0,29^{* *}$ & 1 & $0,32^{* *}$ & $0,22^{*}$ & $0,56^{* *}$ & 0,12 & $-0,35^{* *}$ \\
\hline 5. Busca por Atenção & $0,69^{* *}$ & $0,31^{* *}$ & $0,24^{* *}$ & $0,32^{* *}$ & 1 & 0,17 & $0,42^{* *}$ & 0,13 & $-0,24^{* *}$ \\
\hline $\begin{array}{l}\text { 6. Superficialidade } \\
\text { Interpessoal }\end{array}$ & $0,62^{* *}$ & $0,63^{* *}$ & $0,36^{* *}$ & $0,22^{*}$ & 0,17 & 1 & $-0,07$ & $0,38^{* *}$ & $-0,01$ \\
\hline
\end{tabular}

Nota. *Significativo ao nível de $p=0,05 ; * *$ significativo ao nível de $p=0,001$.

positivas, baixas e moderadas, indiciando que os indivíduos com maiores pontuações na dimensão revisada também tendem a apresentar tendência a oscilações no humor e baixa resiliência (Neuroticismo) e uma busca ativa por relações interpessoais. Em relação à dimensão Agradabilidade é possível observar que todas as magnitudes significativas foram negativas demonstrando que apesar da busca por relações interpessoais, há uma tendência à inadequação e/ou dificuldades nas relações estabelecidas. Também é possível verificar que a dimensão revisada demonstra maior magnitude de correlação com a dimensão Neuroticismo, menor com Extroversão e maior com Agradabilidade, indicando que a nova dimensão avalia mais tipicamente características patológicas/disfuncionais do que a versão original da dimensão. Essa tendência para avaliação de características patológicas era esperada e necessária, na tentativa de lidar com as críticas apresentadas na literatura na avaliação de determinadas características da personalidade (Miller, 2012; Samuel \& Widiger, 2008). Na sequência, na Tabela 3 são apresentadas as correlações com as facetas da dimensão Neuroticismo.

Tabela 3

Correlações com as Facetas de Neuroticismo (NEO-PI-R)

\begin{tabular}{lcccccc}
\hline & Ans. & Raiva & Dep. & Embar. & Impul. & Vuln. \\
\hline $\begin{array}{l}\text { Necessidade de } \\
\text { Atenção (Revisada) }\end{array}$ & $0,243^{* *}$ & $0,333^{* *}$ & $0,253^{* *}$ & 0,166 & $0,450^{* *}$ & $0,349^{* *}$ \\
$\begin{array}{l}\text { Necessidade de } \\
\text { Atenção (Original) }\end{array}$ & $-0,015$ & 0,135 & $-0,069$ & $-0,141$ & 0,178 & $-0,015$ \\
Sedução & & & & & 0,047 \\
Intensidade Emocional & $-0,040$ & 0,167 & $-0,009$ & $-0,043$ & $0,282^{* *}$ & 0,047 \\
Busca por Atenção & $0,393^{* *}$ & $0,511^{* *}$ & $0,467^{* *}$ & $0,331^{* *}$ & $0,486^{* *}$ & $0,443^{* *}$ \\
Superficialidade & $0,341^{* *}$ & $0,330^{* *}$ & $0,315^{* *}$ & $0,264^{* *}$ & $0,349^{* *}$ & $0,406^{* *}$ \\
Interpessoal & $-0,044$ & $-0,097$ & $-0,071$ & $-0,141$ & 0,038 & $-0,030$ \\
\hline
\end{tabular}

Nota. Ans.: Ansiedade; Raiva: Raiva/Hostilidade; Dep.: Depressão; Embar.: Embaraço/Constrangimento; Impul.: Impulsividade; Vuln.: Vulnerabilidade. *significativo ao nível de $p=0,05 ; * *$ significativo ao nível de $p=0,001$. 
Observa-se que a versão revisada da dimensão se correlaciona de maneira mais expressiva com as facetas de Neuroticismo, relacionadas a aspectos disfuncionais (Costa \& McCrae, 2009), o que é adequado para o instrumento, considerando a avaliação de características patológicas da personalidade. Praticamente nenhuma das magnitudes entre Sedução e Superficialidade Interpessoal foi significativa com as facetas de Neuroticismo, contudo, todas entre essas facetas e Intensidade Emocional e Busca por Atenção foram significativas e variaram de baixo para moderado. Esses dados sugerem que pessoas com alta pontuação nas facetas de Neuroticismo tendem a expressar aos outros exageradamente como estão se sentindo e a buscar ativa e explicitamente a atenção dos outros, mas não necessariamente exibem comportamentos de sedução e manipulação ou acreditam estabelecer relações íntimas rapidamente. Na Tabela 4 as correlações com as facetas de Extroversão são apresentadas.

\section{Tabela 4}

\section{Correlações com as Facetas de Extroversão (NEO-PI-R)}

\begin{tabular}{lcccccc}
\hline & Acol & Greg & Assert & Ativ & Busca & Emoções \\
\hline Necessidade de Atenção (Revisada) & $0,261^{* *}$ & $0,258^{* *}$ & 0,110 & $0,258^{* *}$ & $0,433^{* *}$ & $0,247^{* *}$ \\
Necessidade de Atenção (Original) & $0,373^{* *}$ & $0,319^{* *}$ & $0,423^{* *}$ & $0,420^{* *}$ & $0,375^{* *}$ & $0,378^{* *}$ \\
Sedução & 0,138 & 0,053 & $0,204^{*}$ & $0,191^{*}$ & $0,359^{* *}$ & $0,199^{*}$ \\
Intensidade Emocional & 0,070 & 0,040 & $-0,063$ & 0,081 & $0,313^{* *}$ & 0,035 \\
Busca por Atenção & 0,078 & $0,186^{*}$ & 0,000 & 0,095 & 0,160 & $-0,004$ \\
Superficialidade Interpessoal & $0,339^{* *}$ & $0,228^{*}$ & 0,109 & $0,214^{*}$ & $0,229^{*}$ & $0,302^{* *}$ \\
\hline
\end{tabular}

Nota. Acol.: Acolhimento; Greg: Gregarismo; Assert.: Assertividade; Ativ.: Atividade; Busca.: Busca de Sensações; Emoções.: Emoções Positivas.

*significativo ao nível de $p=0,05 ; * *$ significativo ao nível de $p=0,001$.

Na Tabela 4, no que se refere à comparação entre a dimensão original e a revisada, em quase todos os casos as magnitudes entre a versão original e as facetas de Extroversão foram maiores. Considerando que a dimensão Extroversão é voltada tipicamente para características saudáveis da personalidade (Costa \& McCrae, 2009), diferente da dimensão Neuroticismo, esse dado é também um indicador de que a versão revisada da dimensão Necessidade de Atenção apresenta conteúdo mais patológico quando comparada à versão original. Além disso, quatro foram as magnitudes mais expressivas e significativas, sendo elas, Busca de Sensações com Sedução e Intensidade Emocional, indicando que as pessoas que exibem comportamentos de manipulação e sedução e que buscam excessivamente mostrar como estão se sentido são também aqueles que busca excitação e estimulação; e, Superficialidade Interpessoal com Acolhimento e Emoções Positivas, sugerindo que as pessoas que acreditam estabelecer rapidamente relações íntimas são também aquelas que se veem como mais acolhedoras, demonstrando afeto e amizade, bem como tendência a experienciar emoções positivas como alegria e animação (Costa \& McCrae, 2009). Na continuidade, as correlações com as facetas de Agradabilidade são apresentadas na Tabela 5.

Verifica-se que foram duas facetas que se correlacionaram positivamente com praticamente todos os componentes da dimensão revisada Necessidade de Atenção, sendo elas, Franqueza e Modéstia. A faceta Complacência apresentou magnitudes positivas com dois dos quatro componentes. No caso das três facetas, as magnitudes foram negativas, sugerindo que sujeitos com pontuações altas nos componentes da dimensão revisada tendem a não ser leais, tendem a entrar em conflitos interpessoais e a serem vaidosos e pouco humildes (Costa \& McCrae, 2009). No que se refere às demais facetas, somente a magnitude entre Confiança e Superficialidade Interpessoal foi significativa, sugerindo que os sujei- 
tos altos nesse componente tendem a acreditar que as pessoas não são bem intencionadas. $\mathrm{Na}$ sequência as correlações entre os escores totais e componentes da dimensão Necessidade de Atenção e as cinco perguntas do questionário elaborado são apresentadas.

\section{Tabela 5}

\section{Correlações com as Facetas de Agradabilidade (NEO-PI-R)}

\begin{tabular}{lcccccc}
\hline & Conf & Franq & Altr & Comp & Mod & Sens \\
\hline Necessidade de Atenção (Revisada) & $-0,019$ & $-0,489^{* *}$ & $-0,004$ & $-0,347^{* *}$ & $-0,409^{* *}$ & 0,052 \\
Necessidade de Atenção (Original) & 0,051 & $-0,331^{* *}$ & 0,168 & $-0,237^{*}$ & $-0,279^{* *}$ & 0,123 \\
Sedução & 0,004 & $-0,480^{* *}$ & $-0,105$ & $-0,339^{* *}$ & $-0,276^{* *}$ & $-0,035$ \\
Intensidade Emocional & $-0,197^{*}$ & $-0,316^{* *}$ & $-0,065$ & $-0,428^{* *}$ & $-0,239^{* *}$ & 0,067 \\
Busca por Atenção & $-0,117$ & $-0,237^{* *}$ & $-0,119$ & $-0,109$ & $-0,305^{* *}$ & 0,035 \\
Superficialidade Interpessoal & $0,262^{* *}$ & $-0,220^{*}$ & $0,226^{*}$ & $-0,062$ & $-0,274^{* *}$ & 0,088 \\
\hline
\end{tabular}

Nota. Conf.: Confiança; Franq: Franqueza; Altr.: Altruísmo; Comp.: Complacência; Mod.: Modéstia; Sens.: Sensibilidade. *significativo ao nível de $p=0,05 ; * *$ significativo ao nível de $p=0,001$.

\section{Tabela 6}

\section{Correlações com as Perguntas-Critério}

\begin{tabular}{lrrrrrr}
\hline & $\begin{array}{l}\text { Necessidade } \\
\text { de Atenção } \\
\text { (Revisada) }\end{array}$ & $\begin{array}{l}\text { Necessidade } \\
\text { de Atenção } \\
\text { (Original) }\end{array}$ & \multicolumn{1}{c}{ Sedução } & $\begin{array}{c}\text { Intensidade } \\
\text { Emocional }\end{array}$ & $\begin{array}{c}\text { Busca por } \\
\text { Atenção }\end{array}$ & $\begin{array}{c}\text { Superficialidade } \\
\text { Interpessoal }\end{array}$ \\
\hline 1 & $0,292^{* *}$ & $0,301^{* *}$ & $0,212^{*}$ & $0,222^{*}$ & 0,131 & 0,186 \\
2 & $0,244^{*}$ & $0,208^{*}$ & 0,078 & $0,210^{*}$ & $0,267^{* *}$ & 0,120 \\
3 & $0,273^{* *}$ & $0,278^{* *}$ & $0,195^{*}$ & 0,159 & $0,209^{*}$ & 0,111 \\
4 & 0,063 & 0,173 & 0,135 & $-0,013$ & 0,063 & 0,037 \\
5 & 0,170 & $0,190^{*}$ & 0,060 & 0,056 & $-0,012$ & $0,235^{*}$ \\
\hline
\end{tabular}

Nota. 1: Quantas vezes você costuma ir a eventos sociais (festas, etc.) por semana?; 2: Quantas vezes você costuma comprar roupas para você mesmo por semana?; 3: Quanto você se importa com a roupa que usa?; 4: Quantos relacionamentos amorosos você teve?; 5: Quantos amigos (de ambos os sexos) íntimos/próximos você tem? *significativo ao nível de $p=0,05$; **ignificativo ao nível de $p=0,001$.

Em relação às correlações entre as perguntas e os escores totais, as magnitudes foram similares e significativas para a maior parte dos casos, além de positivas, indicando que quanto maior a pontuação dos sujeitos na dimensão Necessidade de Atenção, maior a probabilidade dos comportamentos relacionados às perguntas, quais seja, ir a eventos sociais, comprar roupas, dar importância para a roupa que está usando, número de relações amorosas no passado e número de amigos considerados íntimos. De acordo com Millon (2011), indivíduos com uma tendência ao funcionamento histriônico, intimamente relacionado à dimensão Necessidade de Atenção (Carvalho, 2011), tendem a necessitar estar entre outras pessoas (eventos sociais), preocupar-se com as roupas que usam, ter um histórico permeado por diversas relações amorosas e acreditam ter um número expressivo de amizades íntimas, mais do que as pessoas que não apresentam esse funcionamento.

Especificamente no que diz respeito aos componentes, vale notar que a única magnitude significativa com Superficialidade Interpessoal foi a pergunta número cinco, que se refere ao número de relacionamentos considerados íntimos, o que é coerente considerando que esse componente diz respeito à crença de ser capaz de estabelecer rapidamente relações íntimas. Já o componente Busca por Atenção apresentou 
significância com as perguntas relacionadas à importância com as roupas, o que faz sentido à medida que esse componente está relacionado com uma busca ativa (e comportamentos relacionados) pela atenção dos outros. Tanto Intensidade Emocional quanto Sedução se relacionaram significativamente com a primeira pergunta, tratando da ida à eventos sociais, o que também é coerente já que esses componentes se relacionam com uma necessidade exagerada da pessoa expressar como está se sentido e com o uso de comportamentos de sedução e manipulação, respectivamente. Ainda, as relações aqui encontradas com as perguntas-critério devem ser verificadas e aprofundadas em futuros estudos.

\section{Considerações Finais}

O presente estudo teve como principal objetivo investigar as propriedades psicométricas da versão revisada da dimensão Necessidade de Atenção do IDCP. Especificamente, foram observadas evidências de validade com base na estrutura interna e na relação com variáveis externas, bem como verificou-se os índices de fidedignidade (consistência interna) da dimensão e seus componentes.

Em relação à estrutura interna, sugere-se que as evidências apresentadas foram favoráveis para a nova dimensão Necessidade de Atenção, pois foram encontrados quatro componentes interpretáveis de acordo com as propostas utilizadas como base para a construção da dimensão. Ainda, a partir da comparação do escore total da dimensão original do IDCP e da dimensão revisada, compreende-se que o conteúdo dos novos itens tende a conter características mais patológicas em relação ao conjunto original de itens, o que era esperado e requerido já que o instrumento avalia características patológicas da personalidade. Além disso, é importante ressaltar que cada componente encontrado poderia, da perspectiva psicométrica, ter sido construído com um conjunto maior de itens apresentados. Porém, visou-se restringir ao máximo o número de itens por componente para não impossibilitar o uso do instrumento no ambiente clínico devido a sua extensão.
Os quatro componentes encontrados apresentaram magnitudes de correlação médias e altas entre si, demonstrando que apesar de existir um elemento comum entre eles (construto latente), há também um fator específico atrelado a cada componente. E, no que se refere à consistência interna de cada componente e também da dimensão como um todo, os índices encontrados foram satisfatórios, sobretudo, levando-se em conta o impacto do número pequeno de itens na fórmula subjacente à análise procedida. Ao lado disso, as magnitudes de correlação da nova dimensão com as dimensões e facetas do NEO-PI-R foram no sentido esperado, indicando legitimidade às interpretações realizadas com base no conjunto de itens.

Deve-se ressaltar também as limitações do presente estudo. O primeiro ponto a ser citado refere-se à amostra, tanto ao número de participantes quanto às características dos mesmos. Neste contexto, é importante que futuros estudos se atentem a replicabilidade da estrutura presentemente encontrada em grupos distintos, principalmente as que abrangem pacientes com diagnósticos de transtornos da personalidade. Além disso, uma vez que os componentes presentemente encontrados sejam replicados, é fundamental que os índices de fidedignidade sejam verificados com base nos níveis dos sujeitos no construto latente, por exemplo, via precisão local (Daniel, 1999). Nesse sentido, considerando o escopo do IDCP, faz-se relevante a verificação do uso clínico da nova dimensão.

\section{Referências}

American Educational Research Association, American Psychiatry Association, \& National Council on Measurement in Education. (1999). Standards for Educational and Psychological Testing. New York: American Educational Research Association.

American Psychiatry Association. (2002). Manual Diagnóstico e Estatístico de Transtornos Mentais DSM-IV-TR (4. ed.). Porto Alegre, RS: Artmed.

American Psychiatry Association. (2012). DSM-5 Development. Retrieved February 10, 2013, from http://www.dms5.org 
American Psychiatry Association. (2013). Diagnostic and Statistical Manual of Mental Disorders 5. Washington, DC: Author.

Carvalho, L. F. (2008). Construção e Validação do Inventário Dimensional dos Transtornos da Personalidade (Dissertação de mestrado, Universidade São Francisco, Itatiba, SP, Brasil).

Carvalho, L. F. (2011). Desenvolvimento e Verificação das Propriedades Psicométricas do Inventário Dimensional Clínico da Personalidade (Tese de doutorado, Programa de Pós-Graduação Stricto Sensu em Psicologia, Universidade São Francisco, Itatiba, SP, Brasil).

Carvalho, L. F., \& Primi, R. (2011). Inventário Dimensional Clínico da Personalidade. Manuscrito não publicado, Universidade São Francisco, Itatiba, SP, Brasil.

Carvalho, L. F., Sette, C. P., Primi, R., \& Capitão, C. G. (2013). Revisão da Dimensão Necessidade de Atenção do Inventário Dimensional Clínico da Personalidade (IDCP). Manuscrito submetido para publicação.

Choca, J. P. (2004). Interpretative Guide for the Millon Clinical Multiaxial Inventory III. Washington, DC: American Psychological Association.

Clark, L. A. (1990). Toward a consensual set of symptom clusters for assessment of personality disorder. In J. N. Butcher \& C. D. Spielberger (Eds.), Advances in personality assessment (pp. 243-266). Mahwah, NJ: Lawrence Erlbaum.

Costa, P. T., Jr., \& McCrae, R. (2009). NEO-PI-R - Inventário de Personalidade NEO Revisado Manual. São Paulo, SP: Vetor.

Daniel, M. H. (1999). Behind the scenes: Using new measurement methods on the DAS and KAIT. In S. E. Embretson \& S. L. Hershberger (Eds.), The new rules of measurement: What every psychologist and educator should know (pp. 37-63). Mahwah, NJ: Lawrence Erlbaum.

Embretson, S. E. (1996). The new rules of measurement. Psychological Assessment, 8(4), 341-349.

Hayton, J. C., Allen, D. G., \& Scarpello, V. (2004). Factor retention decisions in exploratory factor analysis: A tutorial on parallel analysis. Organizational Research Methods, 7(2), 191-205.

Hooper, D., Coughlan, J., \& Mullen, M. R. (2008). Structural Equation Modelling: Guidelines for Determining Model Fit. The Electronic Journal of Business Research Methods, 6(1), 53-60.
Krueger, R. F., Derringer, J., Markon, K. E., Watson, D., \& Skodol, A. E. (2011). Initial construction of a maladaptive personality trait model and inventory for DSM-5. Psychological Medicine, 8, $1-12$.

Loureiro, S. R. (2000). Transtornos de Personalidade e avaliação psicodiagnóstica. In F. F. Sisto, E. T. B. Sbardelini, \& R. Primi (Eds.), Contextos e questões da avaliação psicológica (pp. 51-61) São Paulo, SP: Casa do Psicólogo.

Lynam, D. R. (2012). Assessment of maladaptive variants of five factor model traits. Journal of Personality, 80(6), 1593-1614.

Miller, J. D. (2012). Five-Factor Model personality disorder prototypes: A review of their development, validity, and comparison to alternative approaches. Journal of Personality, 80(6), 15651591.

Millon, T. (1993). Personality Disorders: Conceptual distinctions and classification issues. In P. T. Costa \& T. A. Widiger, Personality Disorders and the Five-Factor Model of Personality. Washington, DC: American Psychiatry Association.

Millon, T. (2011). Disorders of Personality: Introducing a DSM/ICD spectrum from normal to abnormal. Mahwah, NJ: Wiley.

Millon, T., \& Davis, R. D. (1996). Disorders of Personality DSM-IV and Beyond. Mahwah, NJ: Wiley.

Millon, T., \& Davis, R. D. (1997). The MCMI--III: Present and Future Directions. Journal of Personality Assessment, 68(1), 69-85.

Millon, T., Grossman, S., \& Tringone, R. (2010). The Millon Personality Spectrometer: A tool for personality spectrum analyses, diagnoses, and treatments. In T. Millon, R. F. Krueger, \& E. Simonsen (Eds.), Contemporary directions in psychopathology: Scientific foundations of the DSM-V and ICD-11 (pp. 391-418). New York: The Guilford Press.

Millon, T., Millon, C. M., \& Davis, R. D. (1994). MCMI-III Manual. Minneapolis, MN: Dicandrien.

Millon, T., Millon, C. M., Meagher, S., Grossman, S., \& Ramanath, R. (2004). Personality Disorders in Modern Life. Mahwah, NJ: Wiley.

Nunnally, J. C. (1978). Psychometric theory. New York: McGraw-Hill. 
Samuel, D. B., \& Gore, W. L. (2012). Maladaptive variants of conscientiousness and agreeableness. Journal of Personality, 80(6), 1669-1696.

Samuel, D. B., \& Widiger, T. A. (2008). A metaanalytic review of the relationships between the five-factor model and DSM-IV-TR personality disorders: A facet level analysis. Clinical Psychology Review, 28(8), 1326-1342.

Shedler, J., \& Westen, D. (2004). Dimensions of personality pathology: An alternative to the five factor model. American Journal of Psychiatry, $161,1743-1754$.

Strack, S., \& Millon, T. (2007). Contributions to the dimensional assessment of personality disorders using Millon's model and the Millon Clinical Multiaxial Inventory (MCMI9-III). Journal of Personality Assessment, 89(1), 56-69.

Tomiatti, M., Gore, W. L., Lynam, D. R., Miller, J. D., \& Widiger, T. A. (2011). A five-factor measure of histrionic personality traits. In N. Gotsiridze-Columbus (Ed.), Psychological assessment. Hauppage, NY: Nova Science.
Watkins, M. W. (2006). Determining parallel analysis criteria. Journal of Modern Applied Statistical Methods, 5(2), 344-346.

Widiger, T. A., \& Costa, P. T., Jr. (2012). Integrating normal and abnormal personality structure: The five factor model. Journal of Personality, 80(6), 1471-1506.

Widiger, T. A., \& Lowe, J. R. (2008). A dimensional model of personality disorder: Proposal for DSM-V. The Psychiatric Clinics of North America, 31(3), 363-378.

Widiger, T. A., \& Trull, T. J. (2007). Place Tectonics in the Classification of Personality Disorder: Shifting to a dimensional model. American Psychologist, 62(2), 71-83.
Recebido: 06/08/2013

$1^{a}$ revisão: 03/09/2013 Aceite final: 06/09/2013 